\title{
Article \\ Lorentz Symmetry Group, Retardation, and Galactic Rotation Curves
}

\author{
Asher Yahalom ${ }^{1,2} \mathbb{B}$ \\ 1 Ariel University, Ariel 40700, Israel; asya@ariel.ac.il \\ 2 Princeton University, Princeton, New Jersey 08543, USA;
}

\begin{abstract}
The general theory of relativity (GR) is known to be invariant under smooth coordinate transformations (diffeomorphism). This group has a subgroup known as the Lorentz group of symmetry which is manifested in the weak field approximation to GR. The dominant operator in the weak field equation of GR is thus the d'Alembert (wave) operator which has a retarded potential solution. Galaxies are huge physical systems having dimensions of many tens of thousands of light years. Thus any change at the galactic center will be noticed at the rim only tens of thousands of years later. Those retardation effects are neglected in present day galactic modelling used to calculate rotational velocities of matter in the rims of the galaxy and surrounding gas. The significant differences between the predictions of Newtonian instantaneous action at a distance and observed velocities are usually explained by either assuming dark matter or by modifying the laws of gravity (MOND). In this paper we will show that taking general relativity seriously without neglecting retardation effects one can explain the radial velocities of galactic matter in the M33 galaxy without postulating dark matter.
\end{abstract}

Keywords: Spacetime symmetry; Relativity of space-time; Lorentz Symmetry Group; Retardation; Galactic Rotation Curves

\section{Introduction}

GR is known to be invariant under smooth coordinate transformations (diffeomorphism). This group has a subgroup known as the Lorentz group of symmetry which is manifested in the weak field approximation to GR. The dominant operator in the weak field equation of GR is thus the d'Alembert (wave) operator which has a retarded potential solution.

From the observational point of view it is well known that GR is verified by many observations. Nevertheless, some observations seems not to fit GR and observed matter. As soon as 1933 Fritz Zwicky realized that the velocities of the Galaxies within the Comma Cluster are way larger than those predicted by the virial theorem in Newtonian theory [1]. He remarked that the amount of matter needed to account for the velocities could be 400 times that of the visible matter. Which led to postulating an unseen form of matter permeating the cluster. Volders in 1959 remarked that stars in the periphery of the neighbor spiral galaxy M33 do not move as expected [2]. The virial theorem in Newtonian Gravity predicts that $M G / r \sim M v^{2}$, that is to say, the rotation curve should increase and at some point bend down and the velocity should drop off as $1 / \sqrt{r}$. In the seventies Rubin and Ford $[3,4]$ showed for a very large sample of spiral galaxies that this behavior is a general feature: velocities at the periphery of the galaxies do not bend down, attain a plateau at some velocity for each galaxy. In what follows we will show that such effects can be deduced from GR if retardation effects are not neglected. The derivation of the retardation force described in previous publications [7-9] is repeated for completeness. However, a fit of the theory to the M33 rotation galaxy is given for the first time. 


\section{General Relativity}

The general theory of relativity is based on two fundamental equations, Einstein equations [10-13]:

$$
G_{\mu \nu}=-\frac{8 \pi G}{c^{4}} T_{\mu \nu}
$$

in which $G_{\mu v}$ is the Einstein tensor, $T_{\mu \nu}$ is the stress-energy tensor, $G \simeq 6.6710^{-11} \mathrm{~m}^{3} \mathrm{~kg}^{-1} \mathrm{~s}^{-2}$ is the gravitational constant and $c \simeq 310^{8} \mathrm{~ms}^{-1}$ is the velocity of light in vacuum (Greek indices are in the range $0-3)$. And the geodesic equation:

$$
\frac{d^{2} x^{\alpha}}{d s^{2}}+\Gamma_{\mu v}^{\alpha} \frac{d x^{\mu}}{d s} \frac{d x^{v}}{d s}=\frac{d u^{\alpha}}{d s}+\Gamma_{\mu \nu}^{\alpha} u^{\mu} u^{v}=0
$$

in which $x^{\alpha}(s)$ are the four dimensional coordinates of the particle in space-time, $s$ is a parameter along the trajectory that for massive particles can be the length of the trajectory, $u^{\mu}=\frac{d x^{\mu}}{d s}$ and $\Gamma_{\mu \nu}^{\alpha}$ is the affine connection (Einstein summation convention is assumed). The stress-energy tensor of matter is usually taken in the form:

$$
T_{\mu v}=\left(p+\rho c^{2}\right) u_{\mu} u_{v}-p g_{\mu v}
$$

In the above $p$ is the pressure, $\rho$ is the density. Lowering and raising indices is done through the metric $g_{\mu \nu}$ and the inverse metric $g^{\mu v}$, that is $u_{\mu}=g_{\mu \nu} u^{v}$. The same metric serves to calculate $s$ :

$$
d s^{2}=g_{\mu \nu} d x^{\mu} d x^{v}
$$

and the affine connection:

$$
\Gamma_{\mu \nu}^{\alpha}=\frac{1}{2} g^{\alpha \beta}\left(g_{\beta \mu, v}+g_{\beta v, \mu}-g_{\mu v, \beta}\right), \quad g_{\beta \mu, v} \equiv \frac{\partial g_{\beta \mu}}{\partial x^{\nu}}
$$

The affine connection serves to calculate the Riemann and Ricci tensors and the curvature scalar:

$$
R_{v \alpha \beta}^{\mu}=\Gamma_{v \alpha, \beta}^{\mu}-\Gamma_{v \beta, \alpha}^{\mu}+\Gamma_{v \alpha}^{\sigma} \Gamma_{\sigma \beta}^{\mu}-\Gamma_{\nu \beta}^{\sigma} \Gamma_{\sigma \alpha,}^{\mu} \quad R_{\alpha \beta}=R_{\alpha \beta \mu}^{\mu}, \quad R=g^{\alpha \beta} R_{\alpha \beta}
$$

which in turn serves to calculate the Einstein tensor:

$$
G_{\alpha \beta}=R_{\alpha \beta}-\frac{1}{2} g_{\alpha \beta} R
$$

Hence matter distribution determines the metric through equation (1) and metric determines trajectories through equation (2) as is well known. Those equations are well known to be symmetric under smooth coordinate transformations (diffeomorphism).

$$
x_{\alpha}^{\prime}=x_{\alpha}^{\prime}\left(x_{\mu}\right)
$$

\section{Linear Approximation of GR}

Except for the extreme cases of compact objects (black holes and neutron stars) and the very early universe (big bang) one need not consider the full non-linear Einstein equation [7]. In most other cases 
of astronomical interest (galactic dynamics included) one can linearize those equations around the flat Lorentz metric $\eta_{\mu v}$ such that ${ }^{1}$ :

$$
\begin{array}{r}
g_{\mu v} \\
\left|h_{\mu v}\right| \ll 1
\end{array}
$$

One than defines the quantity:

$$
\bar{h}_{\mu v} \equiv h_{\mu v}-\frac{1}{2} \eta_{\mu v} h, \quad h=\eta^{\mu v} h_{\mu v}
$$

$\bar{h}_{\mu v}=h_{\mu v}$ for non diagonal terms. For diagonal terms:

$$
\bar{h}=-h \Rightarrow h_{\mu v}=\bar{h}_{\mu v}-\frac{1}{2} \eta_{\mu v} \bar{h}
$$

The general coordinate transformation symmetry of equation (8) has a subgroup of infinitesimal transformations which are manifested in the gauge freedom of $h_{\mu v}$ in the weak field approximation. It can be shown ([10] page 75 exercise 37, see also [11-13]), that one can choose a gauge such that the Einstein equations are:

$$
\square \bar{h}_{\mu v} \equiv \bar{h}_{\mu v, \alpha}{ }^{\alpha}=-\frac{16 \pi G}{c^{4}} T_{\mu v}, \quad \bar{h}_{\mu \alpha,}{ }^{\alpha}=0 .
$$

The d'Alembert operator $\square$ is clearly invariant under the Lorentz symmetry group (another subgroup of the general coordinate transformation symmetry described by equation (8)) of which the Newtonian Laplace operator $\vec{\nabla}^{2}$ is not, but this comes with the price that "action at a distance" solutions are forbidden and only retarded solutions are allowed.

Equation (12) can always be integrated to take the form $[14]^{2}$ :

$$
\begin{aligned}
& \bar{h}_{\mu v}(\vec{x}, t)=-\frac{4 G}{c^{4}} \int \frac{T_{\mu v}\left(\vec{x}^{\prime}, t-\frac{R}{c}\right)}{R} d^{3} x^{\prime}, \\
t \equiv & \frac{x^{0}}{c}, \quad \vec{x} \equiv x^{a} \quad a, b \in[1,2,3], \\
\vec{R} \equiv & \vec{x}-\vec{x}^{\prime}, \quad R=|\vec{R}| .
\end{aligned}
$$

The factor before the integral is small: $\frac{4 G}{c^{4}} \simeq 3.310^{-44}$ hence in the above calculation one can take $T_{\mu v}$ which is zero order in $h_{\alpha \beta}$. Let us now calculate the affine connection in the linear approximation:

$$
\Gamma_{\mu v}^{\alpha}=\frac{1}{2} \eta^{\alpha \beta}\left(h_{\beta \mu, v}+h_{\beta v, \mu}-h_{\mu \nu, \beta}\right) .
$$

The affine connection has only first order terms, hence for a first order approximation of $\Gamma_{\mu v}^{\alpha} u^{\mu} u^{v}$ appearing in the geodesic, $u^{\mu} u^{v}$ is zeroth order. In the zeroth order:

$$
u^{0}=\frac{1}{\sqrt{1-\frac{v^{2}}{c^{2}}}}, u^{a}=\vec{u}=\frac{\frac{\vec{v}}{c}}{\sqrt{1-\frac{v^{2}}{c^{2}}}}, \vec{v} \equiv \frac{d \vec{x}}{d t}, \quad v=|\vec{v}| .
$$

For non relativistic velocities:

$$
u^{0} \simeq 1, \quad \vec{u} \simeq \frac{\vec{v}}{c}, \quad u^{a} \ll u^{0} \quad \text { for } \quad v \ll c .
$$

\footnotetext{
Private communication with the late Professor Donald Lynden-Bell

2 For reasons why the symmetry between space and time is broken see $[15,16]$
} 
Hence, we will not be considering in this paper a post Newtonian approximation in which matter travels at nearly relativistic speeds, but we will be considering the retardation effects and finite propagation speed of the gravitational field. Inserting equation (14) and equation (16) in the geodesic equation we arrive at the approximate form:

$$
\frac{d v^{a}}{d t} \simeq-c^{2} \Gamma_{00}^{a}=-c^{2}\left(h_{0,0}^{a}-\frac{1}{2} h_{00,}^{a}\right)
$$

Let us now look at $T_{\mu v}=\left(p+\rho c^{2}\right) u_{\mu} u_{v}-p g_{\mu v}$. In the current case $\rho c^{2} \gg p$, combining this with equation (16) we arrive at $T_{00}=\rho c^{2}$ while all other components of the tensor $T_{\mu v}$ are significantly smaller. This implies that $\bar{h}_{00}$ is significantly larger than other components of the tensor $\bar{h}_{\mu v}$. Of course one should be careful and not deduce from the different magnitudes of quantities that such a difference exist between their derivatives. In fact by the gauge condition in equation (12):

$$
\bar{h}_{\alpha 0,}{ }^{0}=-\bar{h}_{\alpha a,}{ }^{a} \quad \Rightarrow \bar{h}_{00}{ }^{0}=-\bar{h}_{0 a}{ }^{a}, \quad \bar{h}_{b 0,}{ }^{0}=-\bar{h}_{b a}{ }^{a} .
$$

Hence the zeroth derivative of $\bar{h}_{00}$ (contains a $\frac{1}{c}$ factor) is the same order as the spatial derivative of $\bar{h}_{0 a}$ and like wise the zeroth derivative of $\bar{h}_{0 a}$ (which appears implicitly in equation (17)) is the same order of the spatial derivative of $\bar{h}_{a b}$. However, it is safe to compare spatial derivatives of $\bar{h}_{00}$ and $\bar{h}_{a b}$ and conclude that the former is significantly larger than the later. Using equation (11) and taking the above consideration into account we write equation (17) as:

$$
\frac{d v^{a}}{d t} \simeq \frac{c^{2}}{4} \bar{h}_{00,}{ }^{a} \Rightarrow \frac{d \vec{v}}{d t}=-\vec{\nabla} \phi=\vec{F}, \quad \phi \equiv \frac{c^{2}}{4} \bar{h}_{00}
$$

Thus $\phi$ is a gravitational potential of the motion which can be calculated using equation (13):

$$
\begin{aligned}
\phi & =\frac{c^{2}}{4} \bar{h}_{00}=-\frac{G}{c^{2}} \int \frac{T_{00}\left(\vec{x}^{\prime}, t-\frac{R}{c}\right)}{R} d^{3} x^{\prime} \\
& =-G \int \frac{\rho\left(\vec{x}^{\prime}, t-\frac{R}{c}\right)}{R} d^{3} x^{\prime}
\end{aligned}
$$

and $\vec{F}$ is the force per unit mass. If $\rho$ is static we are in the realm of the Newtonian instantaneous action at a distance theory. However, it is unlikely that $\rho$ is static as a galaxy will attract mass from the intergalactic medium.

\section{Beyond the Newtonian Approximation}

The retardation time $\frac{R}{c}$ which may be a few tens of thousands of years is short with respect to the time that the galactic density changes significantly. This means that we can write a Taylor series for the density:

$$
\rho\left(\vec{x}^{\prime}, t-\frac{R}{c}\right)=\sum_{n=0}^{\infty} \frac{1}{n !} \rho^{(n)}\left(\vec{x}^{\prime}, t\right)\left(-\frac{R}{c}\right)^{n}, \quad \rho^{(n)} \equiv \frac{\partial^{n} \rho}{\partial t^{n}} .
$$

Inserting equation (21) into equation (20) and keeping the first three terms we will obtain:

$$
\begin{aligned}
\phi & =-G \int \frac{\rho\left(\vec{x}^{\prime}, t\right)}{R} d^{3} x^{\prime}+\frac{G}{c} \int \rho^{(1)}\left(\vec{x}^{\prime}, t\right) d^{3} x^{\prime} \\
& -\frac{G}{2 c^{2}} \int R \rho^{(2)}\left(\vec{x}^{\prime}, t\right) d^{3} x^{\prime}
\end{aligned}
$$


The first term will provide the Newtonian potential, the second term does not contribute, the third term will result in the lower order correction to the Newtonian theory:

$$
\phi_{r}=-\frac{G}{2 c^{2}} \int R \rho^{(2)}\left(\vec{x}^{\prime}, t\right) d^{3} x^{\prime}
$$

The total force per unit mass:

$$
\begin{aligned}
\vec{F} & =\vec{F}_{N}+\vec{F}_{r} \\
\vec{F}_{N} & =-\vec{\nabla} \phi_{N}=-G \int \frac{\rho\left(\vec{x}^{\prime}, t\right)}{R^{2}} \hat{R} d^{3} x^{\prime}, \quad \hat{R} \equiv \frac{\vec{R}}{R} \\
\vec{F}_{r} & \equiv-\vec{\nabla} \phi_{r}=\frac{G}{2 c^{2}} \int \rho^{(2)}\left(\vec{x}^{\prime}, t\right) \hat{R} d^{3} x^{\prime}
\end{aligned}
$$

While the Newtonian force $\vec{F}_{N}$ is always attractive the retardation force $\vec{F}_{r}$ can be either attractive or repulsive. Also notice that while the Newtonian force decreases as $\frac{1}{R^{2}}$, the retardation force is independent of distance as long as the Taylor approximation of equation (21) is valid. For short distances the Newtonian force is dominant but as the distances increase the retardation force becomes dominant. Newtonian force can be neglected for distances significantly larger than the retardation distance:

$$
R \gg R_{r} \equiv c \Delta t
$$

$\Delta t$ is the typical duration in which the density $\rho$ changes. Of course for $R \ll R_{r}$ the retardation effect can be neglected and only Newtonian forces should be considered. For large distances $r=|\vec{x}| \rightarrow \infty$ such that $\hat{R} \simeq \frac{\vec{x}}{|\vec{x}|} \equiv \hat{r}$ we obtain:

$$
\vec{F}_{r}=\frac{G}{2 c^{2}} \hat{r} \int \rho^{(2)}\left(\vec{x}^{\prime}, t\right) d^{3} x^{\prime}=\frac{G}{2 c^{2}} \hat{r} \ddot{M}, \quad \ddot{M} \equiv \frac{d^{2} M}{d t^{2}} .
$$

Now as the galaxy attracts intergalactic gas its mass increases thus $\dot{M}>0$, however, as the intergalactic gas is depleted the rate at which the mass increases must decrease hence $\ddot{M}<0$. Thus in the galactic case:

$$
\vec{F}_{r}=-\frac{G}{2 c^{2}}|\ddot{M}| \hat{r}
$$

and the retardation force is attractive.

\section{Dark Matter}

In what circumstances can one confuse retardation with the effect of a non existent "dark matter"? Let us ignore retardation effects and suppose that radial velocities are a result of some mysterious dark matter. In this case we can write for a spherically symmetric mass distribution [17]:

$$
-\frac{v_{c}^{2}}{r} \hat{r}=\vec{F}_{d}=-\frac{G M_{d}(r)}{r^{2}} \hat{r}
$$

$v_{c}$ is the speed of a test particle of constant radius $r$ and $M_{d}(r)$ is the amount of dark matter inside the radius $r$. Comparing equation (28) and equation (27) we see that the "dark matter" mass can be calculated as follows:

$$
M_{d}(r)=\frac{r^{2}|\ddot{M}|}{2 c^{2}}
$$

Now since:

$$
M_{d}(r)=4 \pi \int_{0}^{r} r^{\prime 2} \rho_{d}\left(r^{\prime}\right) d r^{\prime}, \quad \frac{d M_{d}(r)}{d r}=4 \pi r^{2} \rho_{d}(r)
$$


it follows:

$$
\rho_{d}(r)=\frac{|\ddot{M}|}{4 \pi c^{2} r}
$$

This is consistent with observational data of [5] who concluded that the "dark matter" density decreases as $r^{-1.3}$ for M33.

\section{MOND}

Another approach to explaining galactic rotation curves is the claim that either the laws of dynamics (Newton's second law) or the laws of Gravitation (GR) should be modified. This approach championed by Milgrom is denoted "MOND" (Modified Newtonian dynamics) [18]. In one version of this approach Newton's law of gravity is modified:

$$
\vec{F}_{M}=-\frac{G M}{\mu\left(\frac{a}{a_{0}}\right) r^{2}} \hat{r}
$$

In the above $\mu$ is the interpolation function that should be 1 for $a_{0} \ll a$. Let us assume:

$$
\mu\left(\frac{a}{a_{0}}\right)=\frac{1}{1+\left(\frac{a_{0}}{a}\right)^{2}}
$$

If $a_{0} \gg a, \mu \simeq\left(\frac{a}{a_{0}}\right)^{2}$. A test particle revolving in a constant radius will have centrifugal acceleration $a=\frac{v^{2}}{r}$ and thus:

$$
\vec{F}_{M}=-\frac{G M a_{0}^{2}}{v^{4}} \hat{r}
$$

For $v$ constant at a far away distance this expression is similar to the retardation force and thus:

$$
|\ddot{M}|=\frac{2 M a_{0}^{2} c^{2}}{v^{4}} .
$$

Milgrom found $a_{0}=1.210^{-10} \mathrm{~ms}^{-2}$ to be most fitting to the data. The baryonic mass of the M33 galaxy is $210^{40} \mathrm{~kg}$ [6] and the velocity far away from the galaxy is $140,000 \mathrm{~ms}^{-1}$. We thus obtain $|\ddot{M}| \simeq 1.3410^{17} \mathrm{kgs}^{-2}$ and a ratio:

$$
\frac{1}{\Delta t^{2}}=\frac{|\ddot{M}|}{M} \simeq 6.7410^{-24} \mathrm{~s}^{-2} \Rightarrow \Delta t \simeq 3.8510^{11} \mathrm{~s}
$$

This amounts to a typical accumulation acceleration time scale of $\Delta t \simeq 12,206$ years and retardation distance of:

$$
R_{r}=c \Delta t \simeq 12,206 \text { light years. }
$$

In young galaxies the effect of retardation seems insignificant [19], while for older galaxies it seems like somebody is pressing hard on the brakes of mass accumulation. The change of many order of magnitudes in $|\ddot{M}|$ suggest exponential growth (see [9] for a more detailed explanation), hence the following model is suggested:

$$
\begin{gathered}
M(t)=M(0)+\left(\dot{M}(0)-\frac{\ddot{M}(0)}{\alpha}\right) t+\frac{\ddot{M}(0)}{\alpha^{2}}\left(e^{\alpha t}-1\right) \\
\dot{M}(t)=\dot{M}(0)+\frac{\ddot{M}(0)}{\alpha}\left(e^{\alpha t}-1\right) \\
\ddot{M}(t)=\ddot{M}(0) e^{\alpha t}, \alpha>0 .
\end{gathered}
$$


At the present epoch $t=T$ :

$$
\begin{gathered}
\ddot{M}(T)=\ddot{M}(0) e^{\alpha T} \Rightarrow \ddot{M}(0)=\ddot{M}(T) e^{-\alpha T}, \\
\dot{M}(0)-\frac{\ddot{M}(0)}{\alpha}=\dot{M}(T)-\frac{\ddot{M}(T)}{\alpha}
\end{gathered}
$$

This leads to the following expression for galactic mass at $t=T$ :

$$
\begin{aligned}
M(T) & =M(0)+\left(\dot{M}(T)-\frac{\ddot{M}(T)}{\alpha}\right) T \\
& +\frac{\ddot{M}(T)}{\alpha^{2}}\left(1-e^{-\alpha T}\right) .
\end{aligned}
$$

Let us assume that $M(0)=0$ and $\alpha T>>1 \Rightarrow \alpha>>\frac{1}{T} \simeq 2.410^{-18} s^{-1}$. In this case:

$$
M(T) \simeq T\left(\dot{M}(T)-\frac{\ddot{M}(T)}{\alpha}\right) .
$$

Thus we have one equation but two unknowns: $\alpha, \dot{M}(T)$, hence exact values cannot be deduced. However, since $\dot{M}(T)>0$ it follows that:

$$
\dot{M}(T) \simeq \frac{M}{T}-\frac{|\ddot{M}(T)|}{\alpha} \equiv \dot{M}_{L}(T)-\frac{|\ddot{M}(T)|}{\alpha}>0
$$

The linear accumulation rate is: $\dot{M}_{L}(T)=\frac{M}{T} \simeq 4.910^{22} \mathrm{~kg} \mathrm{~s}^{-1}$. The inequality leads to a lower bound on $\alpha$ :

$$
\alpha>\alpha_{c} \equiv \frac{|\ddot{M}(T)|}{\dot{M}_{L}(T)} \simeq 2.7710^{-6} s^{-1}
$$

In the case $\alpha>>\alpha_{c}$ the mass accumulation rate is approximately linear $\dot{M}(T) \simeq \dot{M}_{L}(T)$ and the effect of the second derivative $\ddot{M}(T)$ on the rate of mass accumulation is negligible. However, the effect of $\ddot{M}(T)$ on galactic rotation curves is significant.

\section{A Dynamical Model}

The mass accumulation model described in the previous section is based on a fitting of the second derivative of the galactic mass to the galactic rotation curve. It is intuitively obvious that as mass is accumulated in the galaxy it must be depleted in the intergalactic medium. This is due to the fact that the total mass is conserved still it is of interest to see if this intuition is compatible with a model of gas dynamics. For simplicity we assume that the gas as a barotropic ideal fluid and its dynamics is described by the Euler and continuity equations as follows:

$$
\begin{gathered}
\frac{\partial \rho}{\partial t}+\vec{\nabla} \cdot(\rho \vec{v})=0 \\
\frac{d \vec{v}}{d t} \equiv \frac{\partial \vec{v}}{\partial t}+(\vec{v} \cdot \vec{\nabla}) \vec{v}=-\frac{\vec{\nabla} p(\rho)}{\rho}-\vec{\nabla} \phi
\end{gathered}
$$

In which the pressure $p(\rho)$ is assumed to be a given function of the density, $\frac{\partial}{\partial t}$ is a partial temporal derivative, $\vec{\nabla}$ has its standard meaning in vector analysis and $\frac{d}{d t}$ is the material temporal derivative. We have neglected viscosity terms due to the gas low density. The system is described by cylindrical coordinates $\bar{r}, \theta, z$ in which $z=0$ is the galactic plane. For simplicity we assume axial symmetry, hence all variables are independent of $\theta$. Moreover, the mass influx coming from above and below the galaxy is much more significant as compared to the influx coming from the galactic edge. This is due to the 


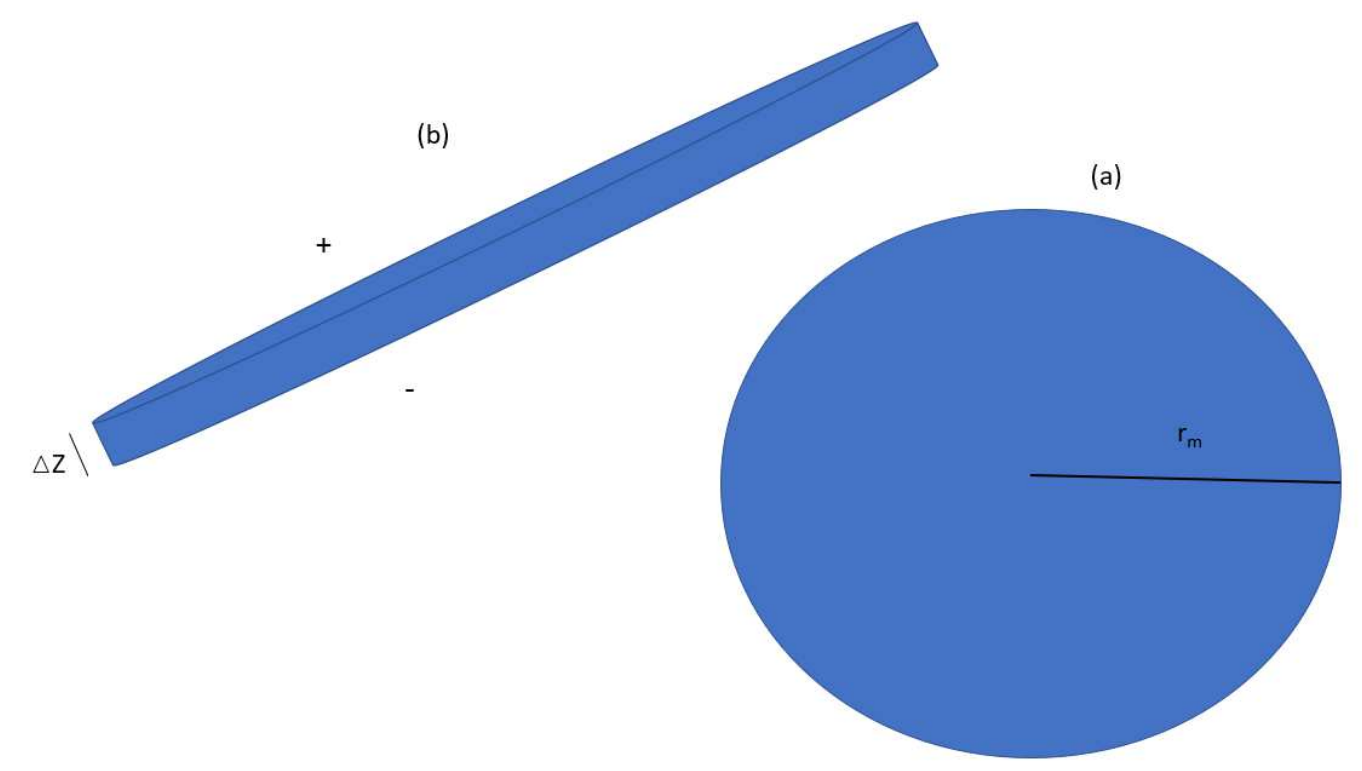

Figure 1. An idealized cylindrical galaxy from different perspectives. (a) from above (b) tilted edge perspective.

large difference of the galaxy surfaces perpendicular to the $\mathrm{z}$ axis compared to the area of its edge. The area of the surface of the galaxy which is perpendicular to the $z$ axis is:

$$
S_{z}=S_{z+}+S_{z-}=\pi r_{m}^{2}+\pi r_{m}^{2}=2 \pi r_{m}^{2}
$$

in which $S_{z}$ is the total surface area of the galaxy perpendicular to the $z$ axis, $S_{z+}$ is the upper area of the surface of the galaxy perpendicular to the $z$ axis, $S_{z-}$ is the lower area of the surface of galaxy perpendicular to the $z$ axis and $r_{m}$ is the galactic radius (see figure 1 ).

The area of the surface of the galactic edge with thickness $\Delta z$ is:

$$
S_{e}=2 \pi r_{m} \Delta z
$$

And thus the ratio of the surfaces area is:

$$
\frac{S_{e}}{S_{z}}=\frac{\Delta z}{r_{m}}
$$

Typical values of $\Delta z$ is about 0.4 kilo parsec and $r_{m}$ is about 17 kilo parsec (for M33) giving an area ratio of about $1 \%$. In such circumstances the edge mass influx is less important and we can assume a velocity field of the form:

$$
\vec{v}=v_{z}(\bar{r}, z, t) \hat{z}+v_{\theta}(\bar{r}, z, t) \hat{\theta} .
$$

$\hat{z}$ and $\hat{\theta}$ are unit vectors in the $z$ and $\theta$ directions respectively. The influx is described schematically in figure 2 . In this case the continuity equation (47) will take the form:

$$
\frac{\partial \rho}{\partial t}+\frac{\partial\left(\rho v_{z}\right)}{\partial z}=0
$$

Defining the quantity:

$$
\gamma \equiv \rho v_{z} \Rightarrow \rho=\frac{\gamma}{v_{z}}
$$

and using the above definition equation (53) takes the form:

$$
\frac{\partial\left(\frac{\gamma}{v_{z}}\right)}{\partial t}+\frac{\partial \gamma}{\partial z}=0
$$




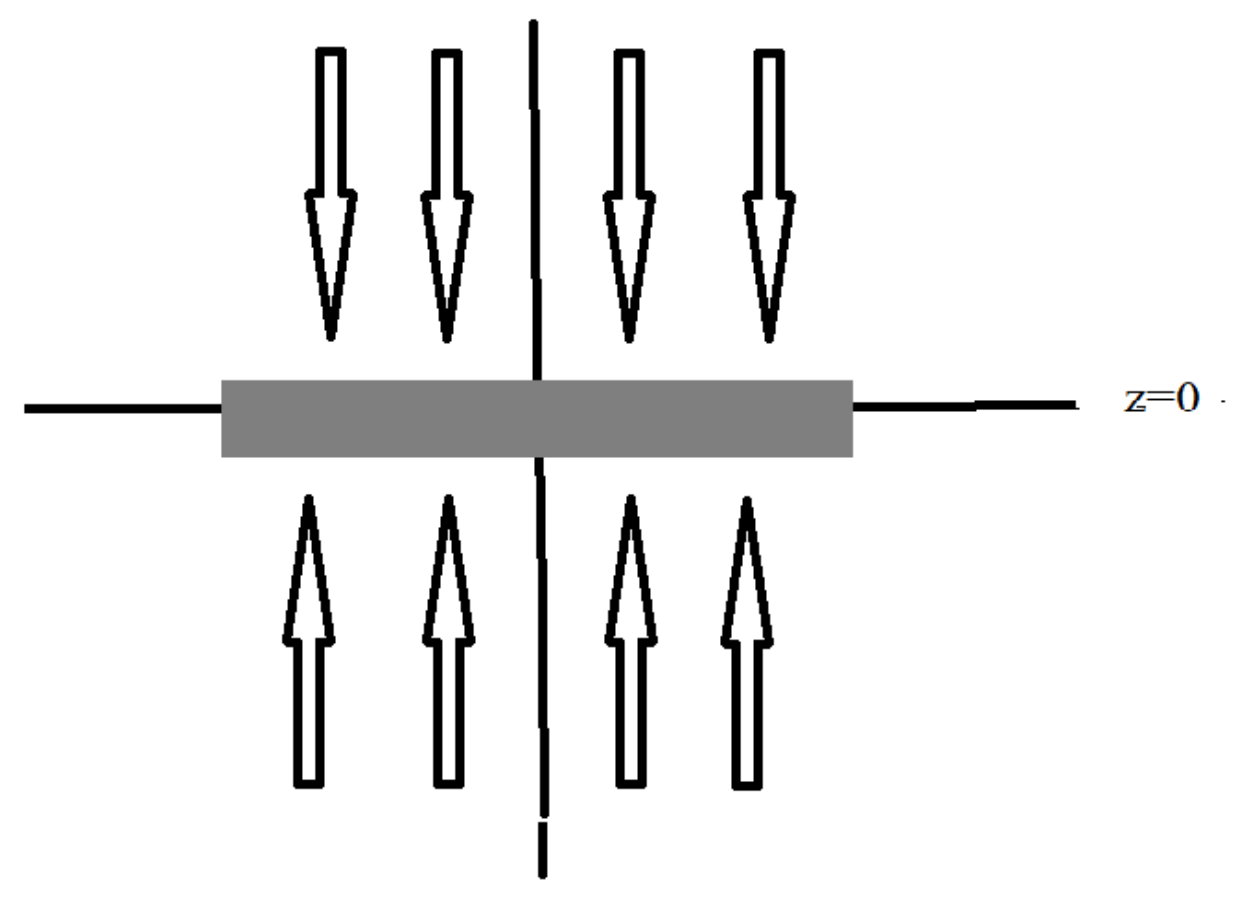

Figure 2. A schematic view of the galactic influx from a side view.

Assuming for simplicity that $v_{z}$ is stationary and defining the auxiliary variable $t_{z}$ :

$$
t_{z} \equiv \int \frac{d z}{v_{z}}
$$

we arrive at the equations:

$$
\frac{\partial \gamma}{\partial t}+\frac{\partial \gamma}{\partial t_{z}}=0
$$

This equation can be solved easily as follows:

$$
\gamma(\bar{r}, z, t)=f\left(t-t_{z}\right), \quad f\left(-t_{z}\right)=\gamma(\bar{r}, z, 0)=v_{z} \rho(\bar{r}, z, 0)
$$

for the function $f(x)$ which is fixed by the density initial conditions and the velocity profile. Let us now turn our attention to the Euler equation (48), for stationary flows it takes the form:

$$
(\vec{v} \cdot \vec{\nabla}) \vec{v}=-\frac{\vec{\nabla} p(\rho)}{\rho}-\vec{\nabla} \phi
$$

According to equation (52) :

$$
\vec{v} \cdot \vec{\nabla}=v_{z} \frac{\partial}{\partial z}+\frac{v_{\theta}}{\bar{r}} \frac{\partial}{\partial \theta}
$$

Now writing equation (59) in terms of its components we arrive at the following equations:

$$
\begin{gathered}
v_{z} \frac{\partial v_{z}}{\partial z}=-\frac{1}{\rho} \frac{\partial p}{\partial z}-\frac{\partial \phi}{\partial z} \\
-\frac{v_{\theta}^{2}}{\bar{r}}=-\frac{1}{\rho} \frac{\partial p}{\partial \bar{r}}-\frac{\partial \phi}{\partial \bar{r}}, \quad\left(\frac{\partial \hat{\theta}}{\partial \theta}=-\hat{\bar{r}}\right) .
\end{gathered}
$$


It is usually assumed that the radial pressure gradients are negligible with respect to the gravitational forces and thus we arrive at the equation:

$$
\frac{v_{\theta}^{2}}{\bar{r}} \simeq \frac{\partial \phi}{\partial \bar{r}}
$$

As for the $z$ component equation it can be easily written in terms of the specific enthalpy $w(\rho)=\int \frac{d P}{\rho}$ in the form:

$$
\frac{\partial}{\partial z}\left(\frac{1}{2} v_{z}^{2}+w(\rho)+\phi\right)=0 \Rightarrow \frac{1}{2} v_{z}^{2}+w(\rho)+\phi=C(r, t)
$$

We recall that $\rho$ depends on $v_{z}$ through equation (54) and equation (58):

$$
\rho(r, z, t)=\frac{\gamma}{v_{z}}=\frac{f\left(t-\int \frac{d z}{v_{z}}\right)}{v_{z}}
$$

As both the specific enthalpy and the gravitational potential are dependent on the density, equation (64) turns into a rather complicated nonlinear integral equation for $v_{z}$. However, many galaxies are flattened structures, hence it can thus be assumed that the pressure $z$ gradients are significant as one approaches the galactic plane. We will thus assume for the sake of simplicity that the pressure gradients balance the gravitational pull of the galaxy and thus $v_{z}$ is just a function of $r$ in which case the convective derivative of $v_{z}$ vanishes. The above assumption holds below and above the galactic plane but not at the galactic plane itself. This suggests the following simple model for the velocity $v_{z}$ (see figure 2):

$$
v_{z}=\left\{\begin{array}{cc}
-\left|v_{z}\right| & z>0 \\
\left|v_{z}\right| & z<0
\end{array}\right.
$$

in which $\left|v_{z}\right|$ is a known function of $\bar{r}$. The velocity field is discontinuous at the galactic plane due to our simplification assumptions, but of course need not be so in reality. We also assume for simplicity that the velocity field $\left|v_{z}\right|$ is constant for $\bar{r}<r_{m}$ and vanishes for $\bar{r}>r_{m}$. According to equation (58) the time dependent density profile is fixed by the density initial conditions. In this section we will deal with the density profile outside the galactic plane and will leave the discussion of the density profile in and near the galactic plane to the next section. We consider an initial density profile as follows:

$$
\begin{aligned}
\rho_{o}(\bar{r}, z, 0) & =\operatorname{re}(z)\left[\rho_{1}(\bar{r})+\rho_{2}(\bar{r}) e^{k|z|}\right] \\
\operatorname{re}(z) & = \begin{cases}1 & |z|<z_{i} \\
0 & |z| \geq z_{i}\end{cases}
\end{aligned}
$$

in which the rectangular function $r e(z)$ keeps the exponential function from diverging. The density profile is depicted in figure 3. We assume that $\rho_{2}$ is negative and thus the density becomes dilute at distances far from the galactic plane. As $v_{z}$ is constant both above and below the galactic plane, $t_{z}=\frac{z}{v_{z}}$ up to a constant. And now it is easy to deduce from equation (58) the functional form of $f(\beta)$ :

$$
f(\beta)=v_{z} r e\left(-v_{z} \beta\right)\left[\rho_{1}+\rho_{2} e^{k\left|v_{z} \beta\right|}\right]
$$

And hence according to equation (69) the time dependent density function for matter outside the galactic plane is obtained:

$$
\rho_{o}(\bar{r}, z, t)=\frac{\gamma}{v_{z}}=\operatorname{re}\left(z-v_{z} t\right)\left[\rho_{1}(\bar{r})+\rho_{2}(\bar{r}) e^{k\left|z-v_{z} t\right|}\right]
$$

The density of matter outside the galactic plane will vanish for $t>t_{m}=\frac{z_{i}}{\left|v_{z}\right|}$, hence we will discuss only the duration of $t<t_{m}$. Let us look at the mass contained in the cylinder defined by the galaxy 


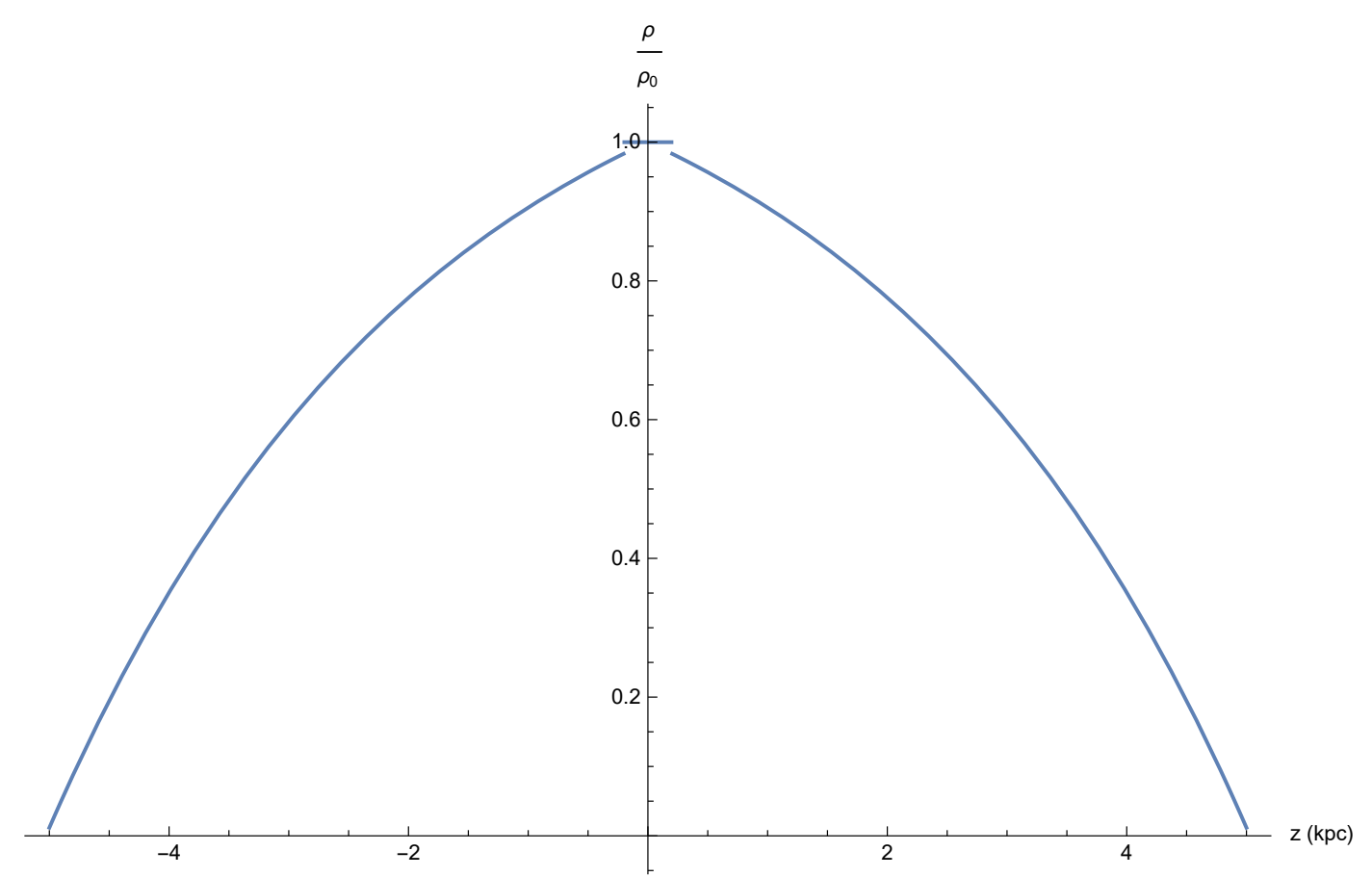

Figure 3. An initial density profile out side the galactic plane. In which $\rho_{0}=\rho_{1}+\rho_{2}, \frac{\rho_{2}}{\rho_{1}}=-0.2$ and $z_{i}=5(\mathrm{kpc})$ and $k=0.32\left(\mathrm{kpc}^{-1}\right)$.

(see figure 4) and let us assume that the total mass in that cylinder is $M_{T}$. Now the mass outside the galactic disk will be:

$$
\begin{aligned}
M_{o}(t) & =2 \pi\left[\int_{-z_{i}}^{-\frac{1}{2} \Delta z} d z \int_{0}^{r_{m}} d \bar{r} \bar{r} \rho_{o}(\bar{r}, z, t)\right. \\
& \left.+\int_{\frac{1}{2} \Delta z}^{z_{i}} d z \int_{0}^{r_{m}} d \bar{r} \bar{r} \rho_{o}(\bar{r}, z, t)\right]
\end{aligned}
$$

Hence the mass in the galactic disk is:

$$
M(t)=M_{T}-M_{o}(t)
$$

And the galactic mass derivatives are:

$$
\dot{M}(t)=-\dot{M}_{o}(t), \quad \ddot{M}(t)=-\ddot{M}_{o}(t)
$$

Inserting equation (69) into equation (70) we may calculate $M_{o}(t)$ :

$$
\begin{aligned}
M_{o}(t) & =2\left[\lambda_{1}\left(z_{i}-\left|v_{z}\right| t-\frac{1}{2} \Delta z\right)\right. \\
& \left.+\frac{\lambda_{2}}{k}\left(e^{k z_{i}}-e^{k\left(\left|v_{z}\right| t+\frac{1}{2} \Delta z\right)}\right)\right]
\end{aligned}
$$

in which:

$$
\lambda_{1} \equiv 2 \pi \int_{0}^{r_{m}} d \bar{r} \bar{r} \rho_{1}(\bar{r}), \quad \lambda_{2} \equiv 2 \pi \int_{0}^{r_{m}} d \bar{r} \bar{r} \rho_{2}(\bar{r}) .
$$

Now calculating the second derivative of $M_{o}(t)$ and using equation (72) leads to the result:

$$
\ddot{M}(t)=-\ddot{M}_{o}(t)=2 k\left|v_{z}\right|^{2} e^{\frac{1}{2} \Delta z k} \lambda_{2} e^{k\left|v_{z}\right| t} .
$$




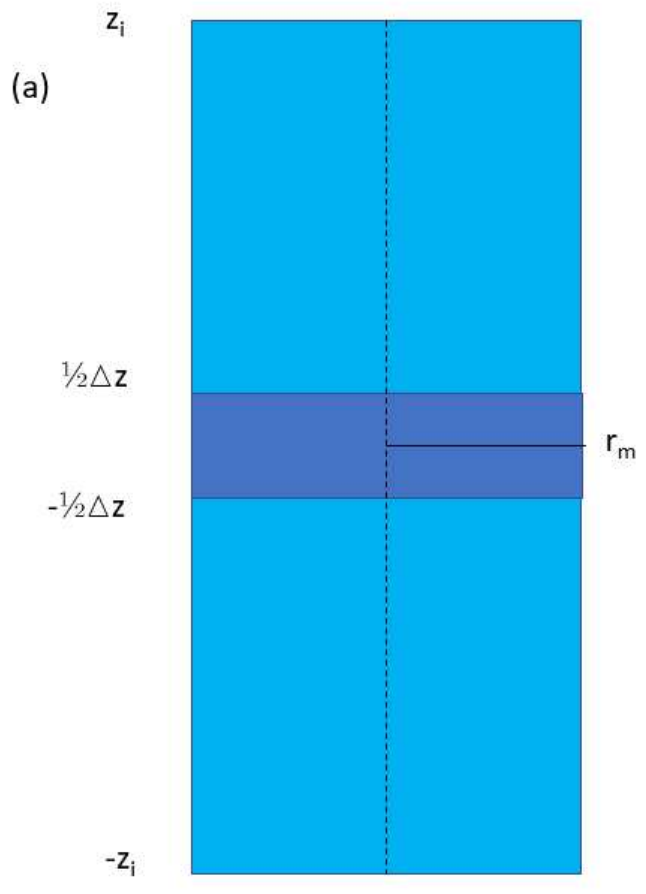

(b)

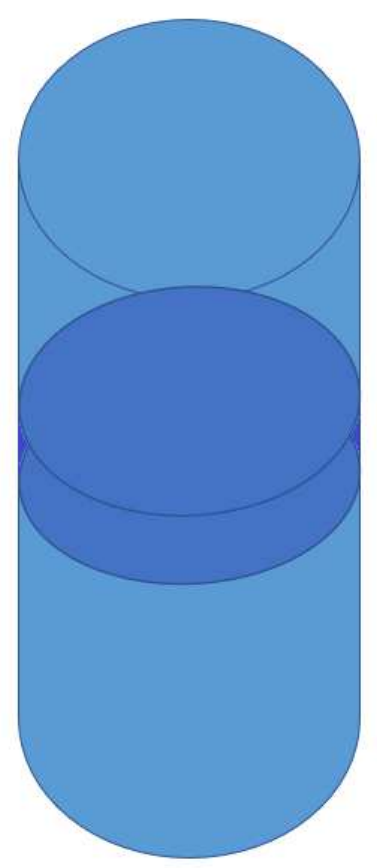

Figure 4. The mass column defined by the galaxy (a) Side view (b) Three dimensional view.

Comparing equation (75) with equation (40) leads to the following identification:

$$
\alpha=k\left|v_{z}\right|, \quad \ddot{M}(0)=2 k\left|v_{z}\right|^{2} e^{\frac{1}{2} \Delta z k} \lambda_{2}
$$

which means that we must have $\lambda_{2}<0$ according to equation (27) in order to assure an attractive force. Next we calculate $\dot{M}(t)$, using equation (72) and equation (73) we will obtain:

$$
\dot{M}(t)=-\dot{M}_{o}(t)=2\left|v_{z}\right| \lambda_{1}+2\left|v_{z}\right| e^{\frac{1}{2} \Delta z k} \lambda_{2} e^{k\left|v_{z}\right| t} .
$$

Hence:

$$
\dot{M}(0)=2\left|v_{z}\right| \lambda_{1}+2\left|v_{z}\right| e^{\frac{1}{2} \Delta z k} \lambda_{2}=2\left|v_{z}\right| \lambda_{1}+\frac{\ddot{M}(0)}{\alpha}
$$

Thus $\lambda_{1}$ is:

$$
\lambda_{1}=\frac{1}{2\left|v_{z}\right|}\left[\dot{M}(0)-\frac{\ddot{M}(0)}{\alpha}\right]
$$

Inserting equation (79) and equation (76) into equation (77) leads back to equation (39). Finally, combining equations $(71,73,76,79)$ and noticing that:

$$
\begin{aligned}
M(0) & =M_{T}-\frac{z_{i}-\frac{1}{2} \Delta z}{\left|v_{z}\right|}\left(\dot{M}(0)-\frac{\ddot{M}(0)}{\alpha}\right) \\
& -\frac{\ddot{M}(0)}{\alpha^{2}}\left(e^{k\left(z_{i}-\frac{1}{2} \Delta z\right)}-1\right)
\end{aligned}
$$

we arrive back at equation (38). Hence the dynamical model presented in this section is compatible with the mass model of the previous one. 


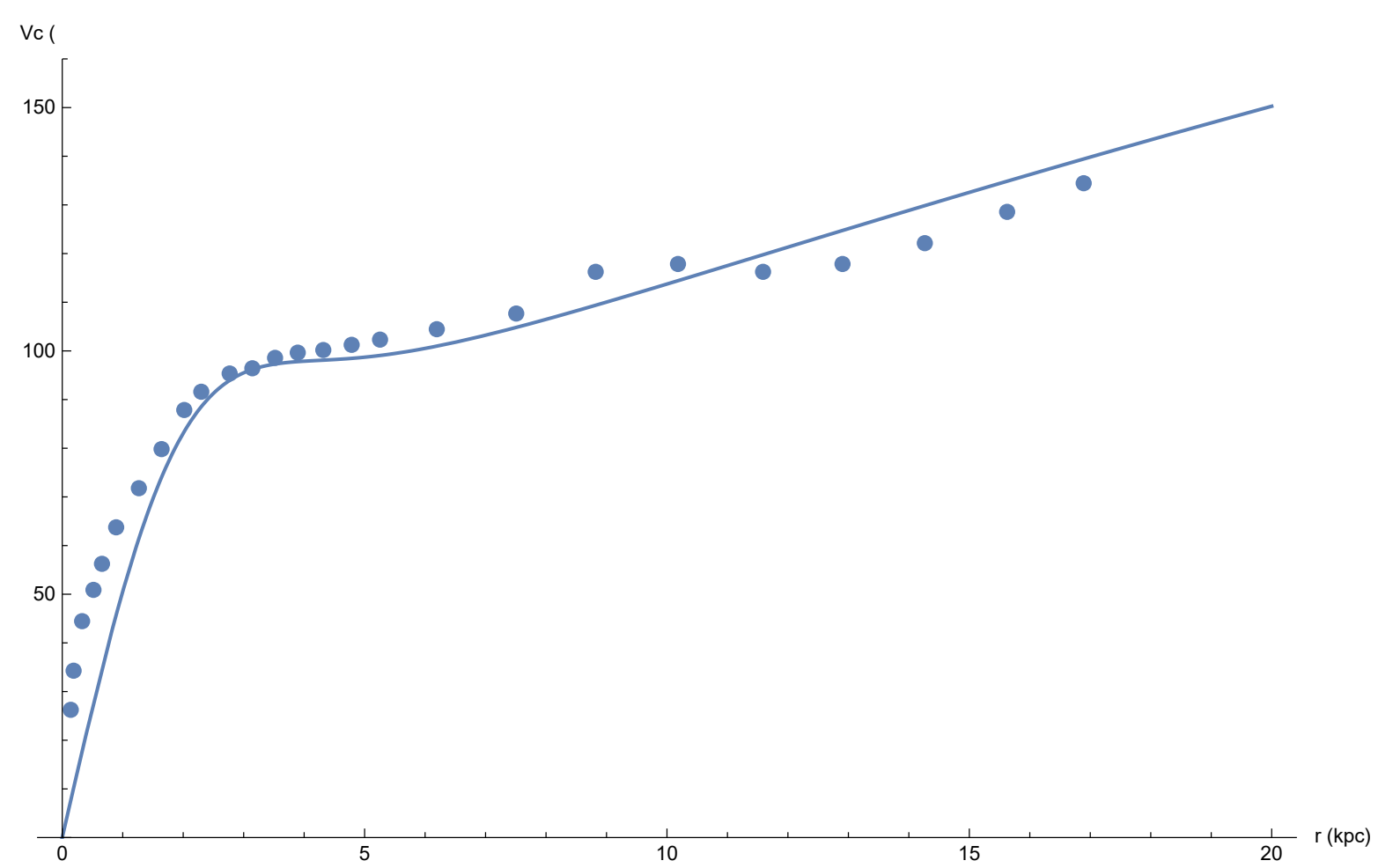

Figure 5. Rotation curve for a sphere with a Gaussian density profile. The author wishes to thank his PhD student Ms. Michal Wagman for supplying the data points of this curve

\section{M33}

Consider a spherical Gaussian density profile:

$$
\rho(r)=\rho_{c} e^{-\frac{r^{2}}{R_{G}^{2}}} .
$$

in which $\rho_{c}$ is the central density and $R_{G}$ the galactic scale. This was fitted to the M33 galactic rotation curve (see figure 5) using the total gravitational force due to both the Newtonian and retardation terms given in equation (24) (Further details can be found in [9]).

The fit is excellent taking into account the crude model.

\section{Conclusion}

The need to satisfy the Lorentz symmetry group prevents the weak field approximation of GR from allowing action at a distance potentials and thus only retarded solutions are allowed. Retardation is manifested more strongly when large distances and large second derivatives are involved.

We show that "dark matter" and "MOND" effects are explained in the framework of standard GR as effects due to retardation without assuming any exotic matter or modifications of the theory of gravity.

What will happen if the mass out side the galaxy is totally depleted? In this case $\ddot{M}=0$ and retardation force should vanish. This was indeed reported recently [19] for the galaxy NGC1052-DF2.

Retardation effects in electromagnetic theory were discussed in [20-22].

\section{Acknowledgement}

The author wishes to thank his PhD student Ms. Michal Wagman for supplying the data points for the rotation curve of the M33 galaxy. 
1. F. Zwicky,"On a New Cluster of Nebulae in Pisces," In: Proc. Natl. Acad. Sci. U S A., May 1937, vol. 23(5), pp. 251-256.

2. L.M.J.S. Volders, "Neutral Hydrogen in M33 and M101," Bull. astr. Inst. Netherl., vol. 14, 323, 1959. V.C. Rubin, W.K. Ford Jr., N. Thonnard, and M.S. Roberts, "Motion of the Galaxy and the Local Group Determined from the Velocity Anisotropy of Distant Sc I Galaxies. I. The Data and II. The Analysis for the Motion," Astrophys. J., vol. 81, 687 and 719, 1976.

3. V.C. Rubin, and W.K. Ford Jr., "Rotation of the Andromeda Nebula from a Spectroscopic Survey of Emission Regions," Astrophys. J., vol. 159, 379, 1970.

4. V.C. Rubin, W.K. Ford Jr., and N. Thonnard, "Rotational Properties of 21 Sc Galaxies with a Large Range of Luminosities and Radii from NGC 4605 (R=4kpc) to UGC 2885 (R=122kpc)," Astrophysical Journal, vol. 238, 471, 1980.

5. E. Corbelli; P. Salucci (2000). "The extended rotation curve and the dark matter halo of M33". Monthly Notices of the Royal Astronomical Society. 311 (2): 441-447. doi:10.1046/j.1365-8711.2000.03075.x.

6. Corbelli E., 2003, MNRAS 342(1): 199-207. DOI: 10.1046/j.1365-8711.2003.06531.x

7. A. Yahalom "The effect of Retardation on Galactic Rotation Curves" Proceedings of the International Association for Relativistic Dynamics (IARD), Merida, Yucatan, Mexico. 4 - 7 June 2018. J. Phys.: Conf. Ser. 1239 (2019) 012006, IOP Publishing https:/ / doi.org/10.1088/1742-6596/1239/1/012006.

8. A. Yahalom "Retardation Effects in Electromagnetism and Gravitation" Proceedings of the Material Technologies and Modeling the Tenth International Conference, Ariel University, Ariel, Israel, August 20 - 24, 2018. (arXiv:1507.02897v2)

9. A. Yahalom "Dark Matter: Reality or a Relativistic Illusion?" Proceedings of Eighteenth Israeli - Russian Bi-National Workshop 2019 "The optimization of composition, structure and properties of metals, oxides, composites, nano and amorphous materials". 17 - 22 February 2019, Ein Bokek, Israel.

10. Narlikar, J. V. (1993). Introduction to Cosmology, Second Edition. Cambridge University Press.

11. A. S. Eddington, "The mathematical theory of relativity" Cambridge University Press (1923)

12. S. Weinberg "Gravitation and Cosmology: Principles and Applications of the General Theory of Relativity" John Wiley \& Sons, Inc. (1972)

13. C. W. Misner, K.S. Thorne \& J.A. Wheeler, "Gravitation" W.H. Freeman \& Company (1973)

14. J. D. Jackson, Classical Electrodynamics, Third Edition. Wiley: New York, (1999).

15. Asher Yahalom "The Geometrical Meaning of Time" Foundations of Physics, Volume 38, Number 6, Pages 489-497 (June 2008).

16. Yahalom, A. (2009). The Gravitational Origin of the Distinction between Space and Time, International Journal of Modern Physics D, Vol. 18, Issue: 14, pp. 2155-2158. Gravity Research Foundation - 2009 Honorable Mention.

17. J. Binney \& S. Tremaine, Galactic Dynamics, Princeton University Press (1987)

18. Milgrom, M. (1983). "A modification of the Newtonian dynamics as a possible alternative to the hidden mass hypothesis". Astrophysical Journal. 270: 365-370. doi:10.1086/161130. Milgrom, M. (1983). "A modification of the Newtonian dynamics - Implications for galaxies". Astrophysical Journal. 270: 371-389. doi:10.1086/161131. Milgrom, M. (1983). "A modification of the Newtonian dynamics - Implications for galaxy systems". Astrophysical Journal. 270: 384. doi:10.1086/161132.

19. Pieter van Dokkum, Shany Danieli, Yotam Cohen, Allison Merritt, Aaron J. Romanowsky, Roberto Abraham, Jean Brodie, Charlie Conroy, Deborah Lokhorst, Lamiya Mowla, Ewan O'Mulligan \& Jielai Zhang "A galaxy lacking dark matter" Nature volume 555, pages 629-632 (29 March 2018) doi:10.1038/nature25767.

20. M. Tuval \& A. Yahalom Newton's Third Law in the Framework of Special Relativity, Eur. Phys. J. Plus 129, 240 (2014). DOI: 10.1140/epjp/i2014-14240-x.

21. M. Tuval and A. Yahalom, Momentum Conservation in a Relativistic Engine, Eur. Phys. J. Plus 131, 374 (2016). DOI: 10.1140/epjp/i2016-16374-1

22. A. Yahalom "Retardation in Special Relativity and the Design of a Relativistic Motor". Acta Physica Polonica A, Vol. 131 (2017) No. 5, 1285-1288. 